\title{
Neutral air turbulence in the mesosphere and associated polar mesospheric summer echoes (PMSEs)
}

\author{
Alireza Mahmoudian ${ }^{1}$, Mike J. Kosch ${ }^{2,3,4}$, Wayne A. Scales ${ }^{5}$, Michael T. Rietveld ${ }^{6}$, and Henry Pinedo ${ }^{7}$ \\ ${ }^{1}$ Institute of Geophysics, University of Tehran, Iran. \\ ${ }^{2}$ Department of Physics, Lancaster University, Lancaster, UK. \\ ${ }^{3}$ South African National Space Agency (SANSA), Hermanus, South Africa \\ ${ }^{4}$ Dept. of Physics and Astronomy, University of the Western Cape, Bellville, South Africa \\ ${ }^{5}$ Bradley Department of Electrical and Computer Engineering, Virginia Tech. \\ ${ }^{6}$ EISCAT Scientific Association, Ramfjordmoen, Norway \\ ${ }^{7}$ Department of Physics and Technology, University of Troms $\varnothing$, Troms $\varnothing$, Norway
}

Correspondence: Alireza Mahmoudian (a.mahmoudian@ut.ac.ir)

\begin{abstract}
This paper presents the first simultaneous four radar frequency observations of the PMSE region under varying neutral air turbulence conditions. Radar frequencies of $8,56,224$, and $930 \mathrm{MHz}$ are used in this study. Three days of experimental observations associated with EISCAT are presented. Numerical simulations of mesospheric dusty/ice plasma associated with the observed radar frequencies are presented. The effect of neutral air turbulence on the generation and strength of plasma density perturbations associated with PMSE using four radar frequencies and in the presence of various dust parameters is investigated. Using the model it is shown that the well-known neutral air turbulence in presence of heavy dust particles, socalled fossil turbulence, can largely explain the observed radar cross-section at four radar frequencies. The effect of initial turbulence amplitude along with dust charging and diffusion in the presence of various dust parameters is investigated using the computational model. Specifically, the response of diffusion to charging time scales, plasma density fluctuation amplitude to the background dusty plasma parameters are discussed. Several key parameters in dusty plasma responsible for the PMSE observations are determined.
\end{abstract}

\section{Introduction}

So-called Polar Mesospheric Summer Echoes PMSEs are very strong coherent radar echoes produced by electron density fluctuations at half the radar wavelength (Bragg scatter condition) in the summer polar mesosphere (80-90 km). While polar mesospheric echoes have been observed in the absence of neutral air turbulence in some cases (Lubken et al., 1993; 2002), theoretical studies by Hill et al. (1999) and Rapp and Lubken (2004) have shown that coupling of the neutral air turbulence with the dusty plasma is the main driving source for PMSE and the associated electron density fluctuations in the mesopause region. Without including dust particles, the small-scale electron density fluctuations produced through coupling of neutral air 
turbulence with electron density fluctuations, diffuse out very fast due to high viscosity effect. Schmidt number $S c$ is defined as a ratio of viscosity $\nu$ to electron diffusion coefficient $D(S c=\nu / D)$ (Lubken et al., 1998). Schmidt number is unity if dust particles are excluded. In other words, the spectrum of electron density fluctuation will have the same cut-off as the neutral air density fluctuations when no dust particles exist. The spectrum of velocity fluctuations in a turbulent medium is scaled with of $k^{-5 / 3}$ where $k$ is the wavenumber. It has been shown that presence of aerosol particles in the mesosphere can increase the Schmidt number to values much greater than unity and extend the viscous cut-off (Cho et al., 1992; Cho and Kelley, 1993; Cho and Rottger, 1997). In addition to the role of charged aerosols in reducing the diffusion timescale of electron density fluctuation, charged aerosol may also result in radar scatter. The so-called dressed aerosol scatter may increase radar scatter above the incoherent scatter and is not dependent on radar wavelength. This theory can explain the observed PMSE using UHF radars. Electron density fluctuations observed in recent in-situ measurements using rocket probes have shown a good agreement with the theory of neutral air turbulence coupling with charged species (Rapp and Lubken, 2004; Lie-Svendsen et al., 2003). Fluctuations in dusty plasma may also be generated by "fossil turbulence" when neutral air turbulence is absent (Cho and Rottger, 1997; Rapp and Lubken, 2004). While velocity field is the driving source for active turbulence, electric field is the generation source for fossil turbulence and fluctuation in plasma and dust densities. It can be shown that electron and ion density fluctuations produced by fossil turbulence are out of phase. The spatial scale of fossil turbulence is typically described by $\omega \ll k c_{n s}$ where $c_{n s}$ is the neutral sound speed (Mahmoudian et al., 2017b). While there are several theories proposed to justify the electron diffusion survived long after the source (neutral air turbulence) has stopped is still an open question in community. La Hoz et al. (2006) used the theoretical model of Hill (1978) which included multipolar diffusion to determine the associated Schmidt numbers. The results required large number of electrons of the order of 10 on the dust particles for enhanced scattering beyond Batchelor scale (Batchelor, 1959). Such a high charge number density may result in electron density bite-out and is far from the range of dust radii observed using sounding rockets in the polar mesosphere (Robertson et al., 2009). The present study is the first attempt to provide a detail study on dusty plasma parameterization of radar echoes in the presence of mesospheric neutral air turbulence and dust particles.

The computational modeling of mesospheric plasma mixed with ice/dust particles which are capable of attracting free ions and electrons has been studied by Lie-Svenson et al. (2003) and Scales (2004). These first studies developed the concept of charging of the mesospheric electrons onto irregular dust density leave electron density "fingerprints" in the form of the electron irregularities that produce PMSE. More simulation work has been done to investigate the temporal evolution of electron irregularities in response to radio wave heating (Mahmoudian et al., 2011; Senior et al., 2014; Mahmoudian et al., 2017a). Very recent work by Mahmoudian et al. (2018) is dedicated to the time evolution of PMSE during enhanced electron density in the mesosphere produced by electron precipitation events. This work demonstrated that the ratio of electron density fluctuation amplitude $\delta n_{e}$ to the plasma density $\left(n_{e}\right)$ plays a critical role in the appearance/disappearance of the layer. The simulation results also revealed that the existence of PMSE is mainly determined by dust radius and dust density.

The coupling of the neutral air turbulence with mesospheric dusty plasma as a generation source of fluctuations in plasma and dust densities as well as electric field is investigated by Mahmoudian et al. (2017b). The impact of the neutral air turbulence wavelength spectrum in the presence of charged dust particles is studied and the extension of the smaller wavelength electron 

with mesospheric dusty plasma was investigated, the steady state situation and the balance of charging and diffusion time scales on the plasma density fluctuation amplitude was overlooked. Therefore, the present study provides the first multifrequency observations of PMSE from $8 \mathrm{MHz}$ up to $930 \mathrm{MHz}$ (corresponding to electron density fluctuation wavelength $40 \mathrm{~m}$ to $16 \mathrm{~cm}$, respectively) as well as time evolution of plasma density fluctuations in numerical modeling of dusty plasma irregularities within associated radar wavelengths. The diffusion and charging processes, electron and ion density variation, and electron density fluctuation amplitude corresponding to the radar echoes are studied for a variety of background dusty plasma parameters. The agreement between the numerical simulations and four radar frequency observations of the PMSE source region simultaneously are presented and discussed.

\section{Experimental observations}

The data collected during 2012 and 2013 research campaigns at EISCAT HF Facility at Ramfjordmoen, Norway $\left(69.6^{\circ} \mathrm{N}\right.$, $\left.19.2^{\circ} \mathrm{E}\right)$, are presented in this paper. $\mathrm{HF}$ radar at $\sim 8 \mathrm{MHz}(7.9 \mathrm{MHz})$ was built using the divided the $\mathrm{HF}$ heater array as the HF radar transmitter. While 10 transmitters on Array 1 where used for heating the ionosphere by radiating vertically in O-mode polarization at $6.77 \mathrm{MHz}$ (an effective radiated power (ERP) of about $600 \mathrm{MW}$ ), simultaneously, the Facility operated in radar mode radiating vertically using two transmitters at $7.953 \mathrm{MHz}$ on Array 1 . This transmission was cycled $48 \mathrm{~s}$ on and $168 \mathrm{~s}$ off starting at 09:00 UT for the case of 10 June 2013 and 26 July 2013. The HF heater alternated between Oand X-mode polarization. The HF radar transmission used a pair of 20 baud complementary codes (10 $\mu$ s bauds and $1.5 \mathrm{~km}$ range resolution). The receiving antenna $\mathrm{O}$-mode gain was $25.2 \mathrm{dBi}$. The O-mode-transmitted pulses received using O-mode polarization separation are used in this study. A complete measurement cycle took $80 \mathrm{~ms}$. It should be noted that the HF radar data presented in this paper are not calibrated for the D-region absorption. The HF radar RCS (radar cross-section) is used in this paper to denote that the effect of ionospheric absorption is not taken into account. Echoes were received digitally and combined in software to produce $\mathrm{O}$ or $\mathrm{X}$ polarization using Array 3 from two orthogonal linear polarizations. The Morro (the MObile Rocket and Radar Observatory) radar at $56 \mathrm{MHz}$ was used to measure the PMSE on the border line of HF and VHF bands (Havnes et al., 2015). The Morro radar was established at EISCAT in 2008 and decommissioned in 2016 (Næsheim et al., 2008). The EISCAT $224 \mathrm{MHz}$ and $930 \mathrm{MHz}$ radars monitored the mesospheric echoes in the same direction. The VHF radars $(56 \mathrm{MHz}$ and $224 \mathrm{MHz}$ ) are not significantly affected by absorption.

Figure 1 shows the simultaneous PMSE observations with 4 radar frequencies. The UHF $(930 \mathrm{MHz})$ radar observation is shown on top panel and was stopped due to technical issue around 11:30 UT. The background electron density of the order of $10^{11} \mathrm{~m}^{-3}$ extends to $80 \mathrm{~km}$ altitude, where the PMSE associated dust/ice particles form, is observed. The VHF radar (224 $\mathrm{MHz}$ ), the second panel from top, also represents the background electron density with a similar magnitude as those observed with UHF radar. The VHF radar also shows simultaneous coherent PMSE that corresponds to electron density fluctuation wavelength of $\sim 67 \mathrm{~cm}$. The third panel shows the radar backscatter from the polar mesospheric clouds at $56 \mathrm{MHz}$. The lower panel shows the HF PMSE at 7.9 MHz which is obtained using the Software Defined Radio Receiver (SDR) radar in 
conjunction with the EISCAT HF facility. As observed in Figure 1, no PMSE is depicted at UHF (corresponding electron density fluctuation wavelength of $16.5 \mathrm{~cm}$ ) during the time of operation. The VHF echoes appear between $\sim 80-87 \mathrm{~km}$ starting around 10:30 UT. The VHF PMSE extends to altitudes lower than $85 \mathrm{~km}$ and becomes much stronger after 12:00 UT. While the overall shape of the PMSE layer looks very similar at 56 and $224 \mathrm{MHz}$, a clear extension and presence of the PMSE layer even before 10:30 UT can be seen in the Morro radar observations. The HF radar has received backscattered echoes from the beginning of the observations at 8:00 UT. The HF echoes show a much stronger pattern and extended over a wider altitude range in comparison with the 56 and $224 \mathrm{MHz}$ echoes. Therefore the observations show consistency with the well developed theory of neutral air turbulence which predicts an extension of turbulence to higher wavenumbers $(k)$ (smaller irregularity wavelength $\lambda$ ) by including the reduced diffusion of fluctuations in the presence of heavy dust particles. This is mainly considered as the source of dusty plasma fluctuations and associated radar echoes (PMSE). Therefore, this will be subsequently investigated quantitavely using numerical simulations. The HF echoes appear at a higher altitude range between $85 \mathrm{~km}$ to $90 \mathrm{~km}$ (8:00 UT-11:00 UT). A slow descent in altitude to the ranges around $85 \mathrm{~km}$ is seen for times after 11:30 UT which is in agreement with the observed 56 and $224 \mathrm{MHz}$ echoes. As discussed in the introduction, the theoretical models are still incapable of characterizing the observed radar echoes in terms of strength and duration with realistic dusty plasma parameters corresponding to the PMSE source region.

Figure 2 shows a similar experimental set up to Figure 1 excluding the $930 \mathrm{MHz}$ radar observations. A stable PMSE layer can be seen from the beginning of the observations at around 09:00 UT on 19 June 2013 at $56 \mathrm{MHz}$ and $224 \mathrm{MHz}$. The overall shape of the layers including the layering phenomenon between 9:50 UT and 11:20 UT as well as the curving structure around 12:30 UT can be clearly seen at both 56 and $224 \mathrm{MHz}$. The comparison between the two frequencies illustrates that the VHF echoes reach a very large amplitude at some times during the experiment and over a narrow altitude range (10:00 UT-11:00 UT). The heating modulation effects including the suppression of radar echoes after the HF heater turn-on and slow recovery to the background unperturbed echoes after a turn-off overshoot effect can be seen in 56 and $224 \mathrm{MHz}$ echoes (Havnes et al., 2015). Although the signature of HF pump modulation can be seen in the data, the overall intensity of the echoes at both frequencies remains intact. La Hoz et al. (2006) have also shown that a large increase in the electron temperature does not affect mesospheric neutral turbulent state as an external driver for PMSE. This will make it possible to use the data presented here as a benchmark for comparison with the numerical simulations presented in the following section in order to investigate neutral air turbulence effects on the formation of plasma irregularities. The overall range of presence in altitude and intensity of $56 \mathrm{MHz}$ echoes is higher in comparison with the $224 \mathrm{MHz}$ echoes. The HF echoes are much stronger in comparison with 56 and $224 \mathrm{MHz}$, and cover a much wider altitude range. As can be seen in Figure 2, the $8 \mathrm{MHz}$ echoes start around $84 \mathrm{~km}$ and extend to $\sim 91 \mathrm{~km}$. Towards the end of experiment the lower bound of HF echoes reaches $\sim 80 \mathrm{~km}$ (after 11:00 UT). No layering structure is seen in $8 \mathrm{MHz}$ echoes. It's noteworthy that the HF data are not calibrated for the lower ionospheric absorption which is appeared to be of the order of a few dBs (Senior et al., 2014).

Figure 3 includes the PMSE at all 4 radar frequencies available at EISCAT on 26 July 2013 between 9:00 UT to 13:00 UT. The UHF (930 MHz) echoes imply the background mesospheric conditions were quiet during this experiment. The UHF data shows a weak PMSE layer around $82 \mathrm{~km}$ starting at 11:40 UT to 12:00 UT. All other three radars used in the experiment show 
the existence of PMSE at various strength. The $224 \mathrm{MHz}$ radar show a discontinuous echoes center around $84 \mathrm{~km}$. While the lower VHF PMSE layers continue to 11:30 UT, a much stronger and double $224 \mathrm{MHz}$ echo layer appear between 85 to $87 \mathrm{~km}$. Similar behavior of layering structure to Figure 2 is observed at 56 and $224 \mathrm{MHz}$, although the $8 \mathrm{MHz}$ radar shows a continuous layer of echoes with altitude. Overall intensity of HF echoes is much higher than 56 and $224 \mathrm{MHz}$. Such a difference can be seen for $224 \mathrm{MHz}$ in comparison with $930 \mathrm{MHz}$. This effect will be investigated throughout this paper using numerical simulations of the perturbed mesospheric dusty plasma with different background parameters. This study aims at elucidating on the theory of neutral air turbulence as the source of mesospheric plasma density fluctuations responsible for coherent radar echoes and in the proximity of dust particles. The size and density of dust particles along with the initial amplitude of irregularities within dust density as a result of coupling with neutral turbulence will be investigated.

\section{Numerical simulations}

One way of understanding the response of the electron density fluctuation amplitude to the background dust plasma parameters is to use the analytical expression for the timescale of physical processes that actually affect the density fluctuations. In general two processes of charging (electron/ion attachment to the dust particles) and plasma density diffusion determine the steady state amplitude of fluctuations in the plasma density. The diffusion process tends to smooth out irregularities and can be approximated for the natural PMSE layer $\left(T_{e} / T_{i}=1\right)$ by (Chen and Scales, 2005; Mahmoudian et al., 2011):

$\tau_{\text {diff }} \approx \nu_{\text {in }}\left(\frac{\lambda_{\text {irreg }}}{2 \pi v_{\text {thi }}}\right)^{2} \frac{1}{\left(1+\left(1+\frac{z_{d 0} n_{d 0}}{n_{e 0}}\right)\right)}$

where $\nu_{i n}, z_{d 0}, n_{e,(d)} \lambda_{i r r e g}$ and $v_{t h i}$ are the ion-neutral collision frequency, charge density on the dust particles, electron (dust) density, electron density irregularity wavelength and ion thermal velocity, respectively. According to the theoretical expression of diffusion time scale this is mainly due to the dependency of diffusion time scales on $\lambda_{\text {irreg }}$.

The timescale for electron attachment onto the dust is approximated by

$\tau_{c h g} \approx \frac{1}{\sqrt{8 \pi} r_{d}^{2} v_{t e 0} e^{-4.1} n_{d 0}}$

While such simple theoretical expressions predict the dependency of radar echoes on the background dusty plasma parameters, they are unable to predict the time evolution nor steady state amplitude of irregularities $\left(\delta n_{e}^{2}\right)$ responsible for radar echoes. Therefore, the computational model is incorporated in this paper to study the characteristics of radar echoes with respect to the dusty plasma parameters. As can be seen in Eq. (1), the diffusion timescale depends on the $\lambda_{\text {irreg }}$. The numerical simulations presented in this paper are associated with radar frequencies slightly different from the observations due to a limitation of the model having a discrete spatial grid. The difference is of the order of a few centimeters in fluctuation wavelength, and are not expected to change the physical processes and the results. 

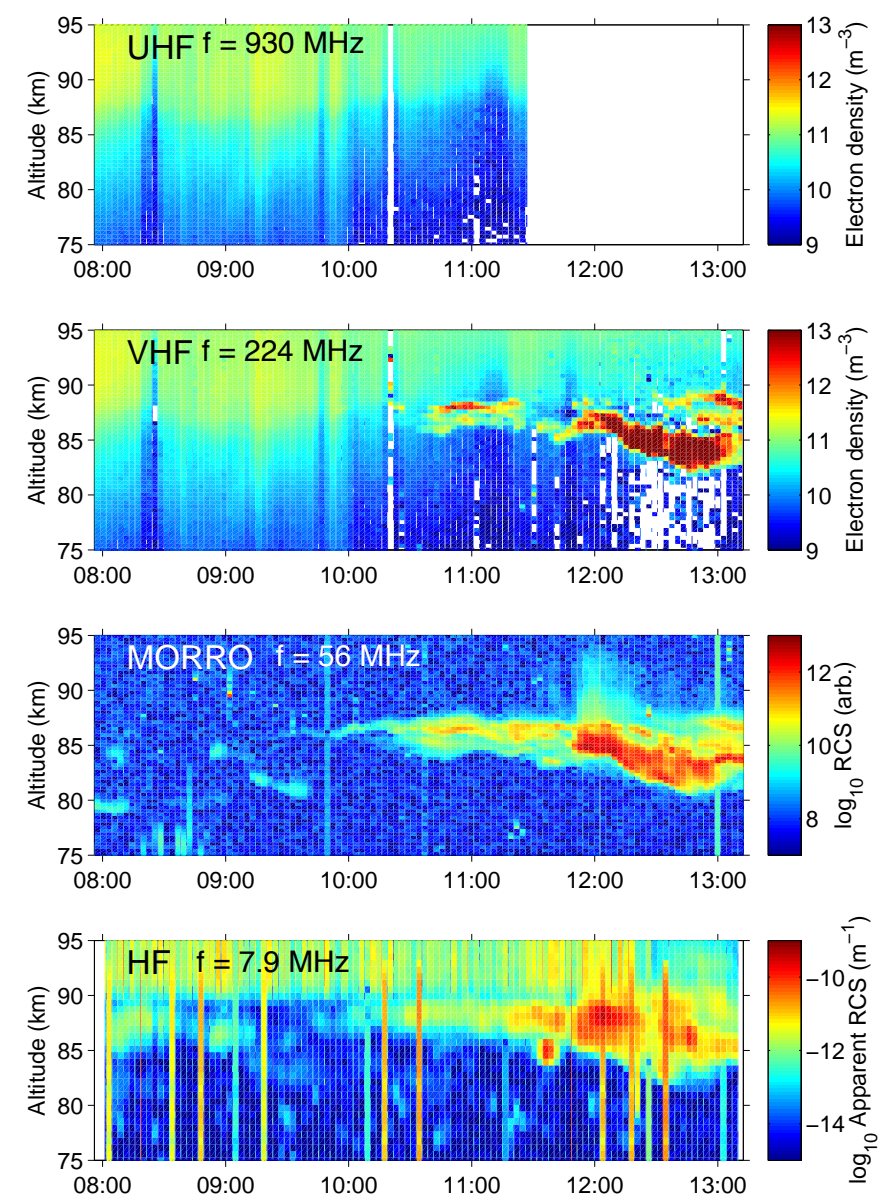

Figure 1. Simultaneous PMSE observations using $930 \mathrm{MHz}, 224 \mathrm{MHz}, 56 \mathrm{MHz}$, and $8 \mathrm{MHz}$ radars, respectively from the top to the bottom, at EISCAT. The experiment was conducted on 12 July 2012 between 8:00 UT to 13:10 UT.

In order to investigate the time evolution of fluctuations in the plasma density due to coupling with neutral air turbulence, the dusty plasma model originally developed at Virginia Tech is used in this study (Scales, 2004; Mahmoudian et al., 2011). The model treats the plasma as a fluid including an arbitrary number of charged particles, neutral particle species and dust/aerosol particles which are modeled as particle in cell (PIC) (Birdsall and Langdon, 1991). Continuous charging model based on the Orbital-Motion-Limited (OML) approach has been used for the time varying charge on the dust particles. The summer mesopause temperature for both ions and electrons is taken to be $T_{e}=T_{i}=150 \mathrm{~K}$. The ion-neutral collision frequency is of order of $10^{5} \mathrm{~s}^{-1}$. The electron density is assumed to be $2 \times 10^{9} \mathrm{~m}^{-3}$. The numerical simulations presented in this paper includes 


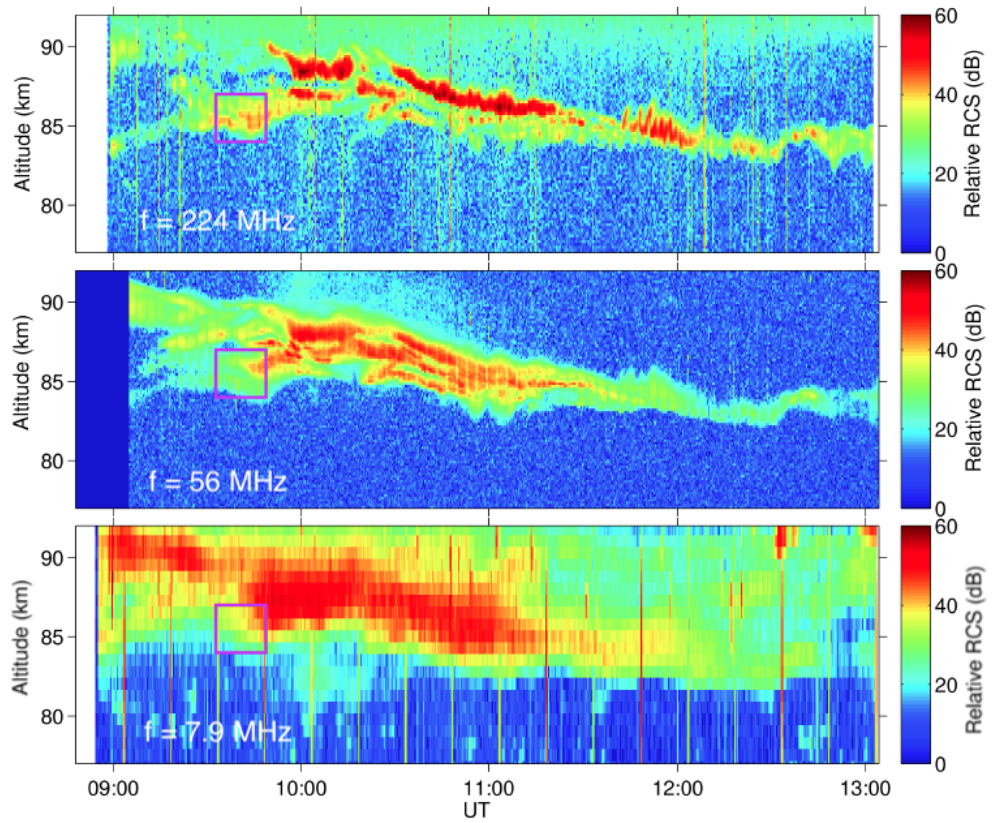

Figure 2. Simultaneous PMSE observations using $224 \mathrm{MHz}$ (top), $56 \mathrm{MHz}$ (middle), and $8 \mathrm{MHz}$ (bottom) radars at EISCAT. The experiment was conducted on 19 June 2013 between 9:00 UT to 13:00 UT. The wavelength associated with each panel correspond to the radar frequency is shown. The electron density fluctuation wavelength are at the half of radar wavelength (Bragg scattering condition).

a wide range of dusty plasma parameters such as small and large dust particles as well as various dust densities. This is mainly to test the proposed theories such as fossil turbulence in order to explain the observed PMSE at a small wavelength where the original neutral turbulence has disappeared due to kinematic viscosity and turbulence energy dissipation. A close comparison of computational results with the radar observations will be discussed in the following section to determine the most important dusty plasma parameters responsible for the long duration of small scale plasma density fluctuations in the PMSE region.

The time variation of 3 parameters in the simulations are are considered in this study. These parameters include electron density fluctuation amplitude squared $\left(\delta n_{e}^{2}\right)$ equivalent to radar echoes, diffusion to charging time scales $\left(\tau_{d i f f} / \tau_{c h g}\right)$, and time evolutions of electron and ion densities, which corresponds to the electron density depletion as a result of charging on to dust particles. The model ran to reach the steady state condition assuming the formation of dust/ice particle in the vicinity of mesospheric plasma. The $\tau_{d i f f} / \tau_{c h g}$ value is a critical parameter that governs the time evolution and determines the steady state amplitude of electron density fluctuation amplitude (radar backscattered amplitude). The electron density is studied in order to investigate the electron depletion level as a parameter that also influences the backscattered radar signal as well as the ion density to monitor the quasi-neutrality condition in the dusty plasma. The electron density fluctuation amplitude starts to increase initially due to dominant charging process. As the diffusion time scale becomes comparable to the charging timescale, it slows down the increase of electron irregularity amplitude until they reach steady state situation. Such a condition is achieved when the dust particles are saturated by the electron/ion charging currents. According to $\tau_{d i f f} / \tau_{c h g}$, for small dust particles 


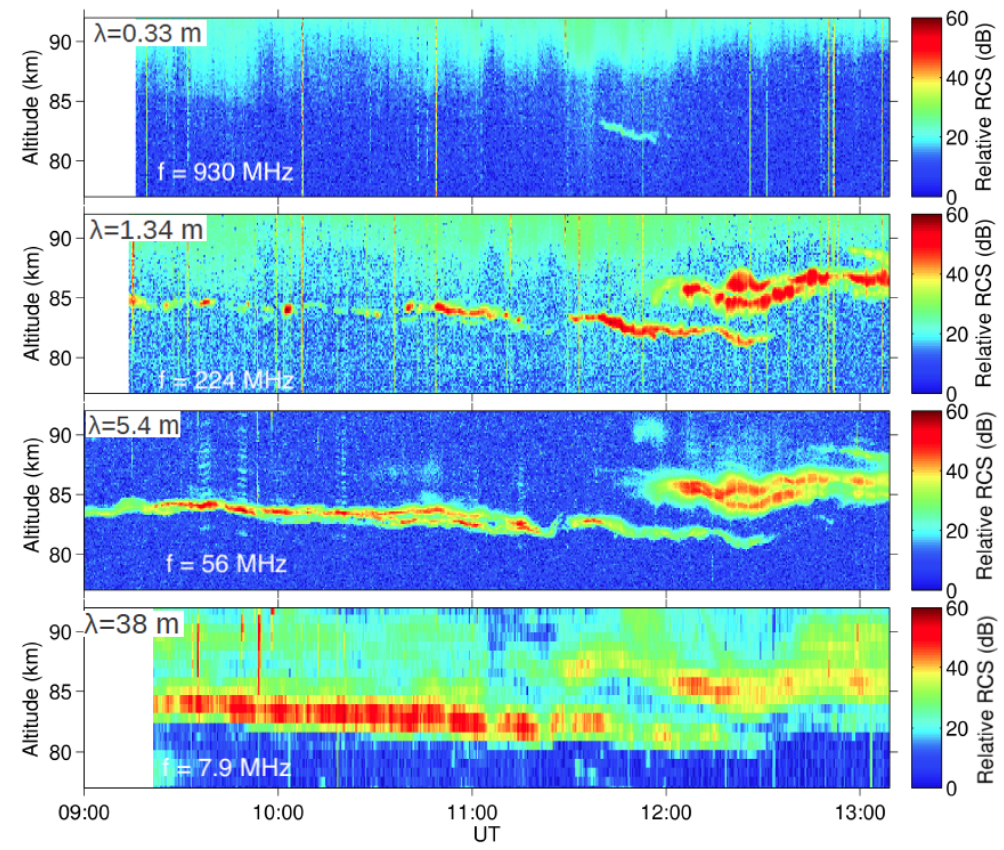

Figure 3. Simultaneous PMSE observations using $930 \mathrm{MHz}, 224 \mathrm{MHz}, 56 \mathrm{MHz}$, and $8 \mathrm{MHz}$ radars, respectively from the top to the bottom, at EISCAT. The experiment was conducted on 26 July 2013 between 9:00 UT to 13:00 UT.

of $3 \mathrm{~nm}$ and $10 \mathrm{~nm}$ this condition is satisfied within $180 \mathrm{sec}$ and $\sim 80 \mathrm{sec}$, respectively (Figures 4-7). The initial fluctuation amplitude in the dust density $\left(\delta n_{d} / n_{d}\right)$ which is caused by neutral air turbulence produces a footprint in the background density. As will be discussed shortly, increase of $\delta n_{d} / n_{d}$ from 0.5 to 1 causes a substantial increase in $\delta n_{e}^{2}$. Therefore, this parameter has a great impact on the corresponding radar echoes in different bands. The black, red, blue and green colors are used in Figures 4-7 for the radar frequencies 7.3 MHz, $58 \mathrm{MHz}, 234 \mathrm{MHz}$ and $930 \mathrm{MHz}$, respectively.

As can be seen in Figures 4 and 5, for smaller dust radius of $3 \mathrm{~nm}$ the ion density reaches values higher than the equilibrium condition due to low ion charging rate with respect to electron charging and in comparison with the production rate (photoionization). For larger dust particles of $10 \mathrm{~nm}$, the ion density reduces as a result of ion charging process on to the dust particles. Such a reduction is of the order of 2 and $15 \%$ for dust densities of $10 \%$ and $70 \%\left(n_{d} / n_{e 0}\right)$, respectively. One of the main characteristics that can be seen in the electron density depletion at various wavelengths (radar frequencies) is the symmetrical behavior. The average electron density (total depletion) is independent of radar frequency. The depletion level reaches $\sim$ $20 \%, 35 \%, 12 \%$, and 70\%, in Figures 4-7, respectively. A very small discrepancy in the averaged electron density reduction is seen for large dust particles of $10 \mathrm{~nm}$ and high dust density of $70 \%\left(n_{d} / n_{e 0}\right)$ (Figure $7 \mathrm{c}$ ). This is of the order of $2 \%$, that is negligible. This behavior excludes the idea that electron density depletion could contribute directly in the PMSE. Such effects prove the coherent nature of the PMSE. Figures $4 \mathrm{a}, 5 \mathrm{a}, 6 \mathrm{a}$, and $7 \mathrm{a}$ show the time evolution of $\delta n_{e}^{2}$, which is proxy for the radar 
echoes. The right panel shows the corresponding radar echoes (amplitude $\log _{10} N_{e} \mathrm{~m}^{-3}$ ). The values are calculated assuming $\delta n_{d} / n_{d}=100 \%$. For the solid curves associated with $\delta n_{d} / n_{d}=50 \%$, the values should be subtracted by $\sim-0.7 \mathrm{~dB}$.

Another unique feature observed in the numerical simulations is the temporal evolution of $\delta n_{e}^{2}$. While for low densities (independent of dust radius) a slow increase in $\delta n_{e}^{2}$ amplitude to steady state value is observed (Figures 4a and 6a), for higher dust densities an overshoot effect and then a slow decrease to the steady state value is seen (Figures 5a and 7a). Such behavior is mainly due to high initial charging process associated with more dust particles present in the plasma. This phenomena can be clearly seen in the $\tau_{d i f f} / \tau_{c h g}$ plot for $r_{d}=10 \mathrm{~nm}, n_{d} / n_{e 0}=70 \%$ (Figure $7 \mathrm{~b}$ ). According to this figure, a sharp decrease in $\tau_{\text {diff }} / \tau_{\text {chg }}$ for values below $10^{-6}$ around 40 sec shows the dominant diffusion process that results in a slow decrease in $\delta n_{e}^{2}$ amplitude.

The temporal evolution of electron density fluctuation amplitude associated with four parameter regimes are examined in this paper. The four parameter set-ups are chosen based on the major parameters that could affect the radar echoes. According to the charging and diffusion timescales that determine the steady sate value of radar echoes (electron density fluctuation amplitude at different wavelengths), dust radius $r_{d}$, dust density $n_{d}$, and dust density fluctuation amplitude $\delta n_{d}$ are considered to vary in the simulations. We assumed the heating modulation by the EISCAT HF transmitter has a minimal effect on the time evolution of radar echoes over 4 hours of observations $\left(T_{e} / T_{i}=1\right)$. The $\delta n_{e}^{2}$ and the corresponding radar echoes increases drastically with increasing $n_{d}$. A comparison of the results associated with the same dust radius (for example $3 \mathrm{~nm}$ ) shows an increase of $\delta n_{e}^{2}$ by a factor of 2 for all radar frequencies (irregularity wavelengths) as the $n_{d}$ increases from $90 \%$ to $150 \%$. A general empirical relationship for increase of $\delta n_{e}^{2}$ by a factor of $\sim\left(n_{d 2} / n_{d 1}\right)^{(6 / 5)}$ is obtained based on the computational results for the same dust radius.

One of the main features that has been observed in the numerical results is that the $\tau_{\text {diff }} / \tau_{c h g}$ for the radar frequencies 234 $\mathrm{MHz}$ and $936 \mathrm{MHz}$ is on the same order. As can be seen in Figures 4-7, changing the background dusty plasma parameters may vary the $\delta n_{e}^{2}$ which corresponds to the radar echoes, but the diffusion and charging time scales stay about the same. A small difference in the estimated amplitude of $\delta n_{e}^{2}$ for similar dust parameters associated with $224 \mathrm{MHz}$ and $930 \mathrm{MHz}$ radars contradicts the observational data. Considering that the observed UHF echoes is expected to be in a similar altitude range as other radar frequencies, the background dust parameters such as $r_{d}$ and $n_{d}$ should be the same in the simulations. Therefore, a close comparison between the $\delta n_{e}^{2}$ for $234 \mathrm{MHz}$ and $936 \mathrm{MHz}$ from the simulations with the same $r_{d}$ and $n_{d}$, reveals a

215 very small difference in the amplitude. Such numerical prediction requires the observation of PMSE at $930 \mathrm{MHz}$. This is not consistent with the observations. The numerical estimation of $\delta n_{e}^{2}$ for reduced $\delta n_{d} / n_{d}$ values from 1 to 0.5 shows that the expected radar echoes are estimated to decrease at least $1 \mathrm{~dB}$. This could explain the non observation of UHF PMSE due to dissipation of fluctuations at such a short wavelength.

\section{Summary and conclusion}

The previous studies have shown that the small-scale electron density fluctuations produced through coupling of neutral air turbulence and charging process of electrons onto irregular dust density may be extended to higher $k$ values (corresponding 

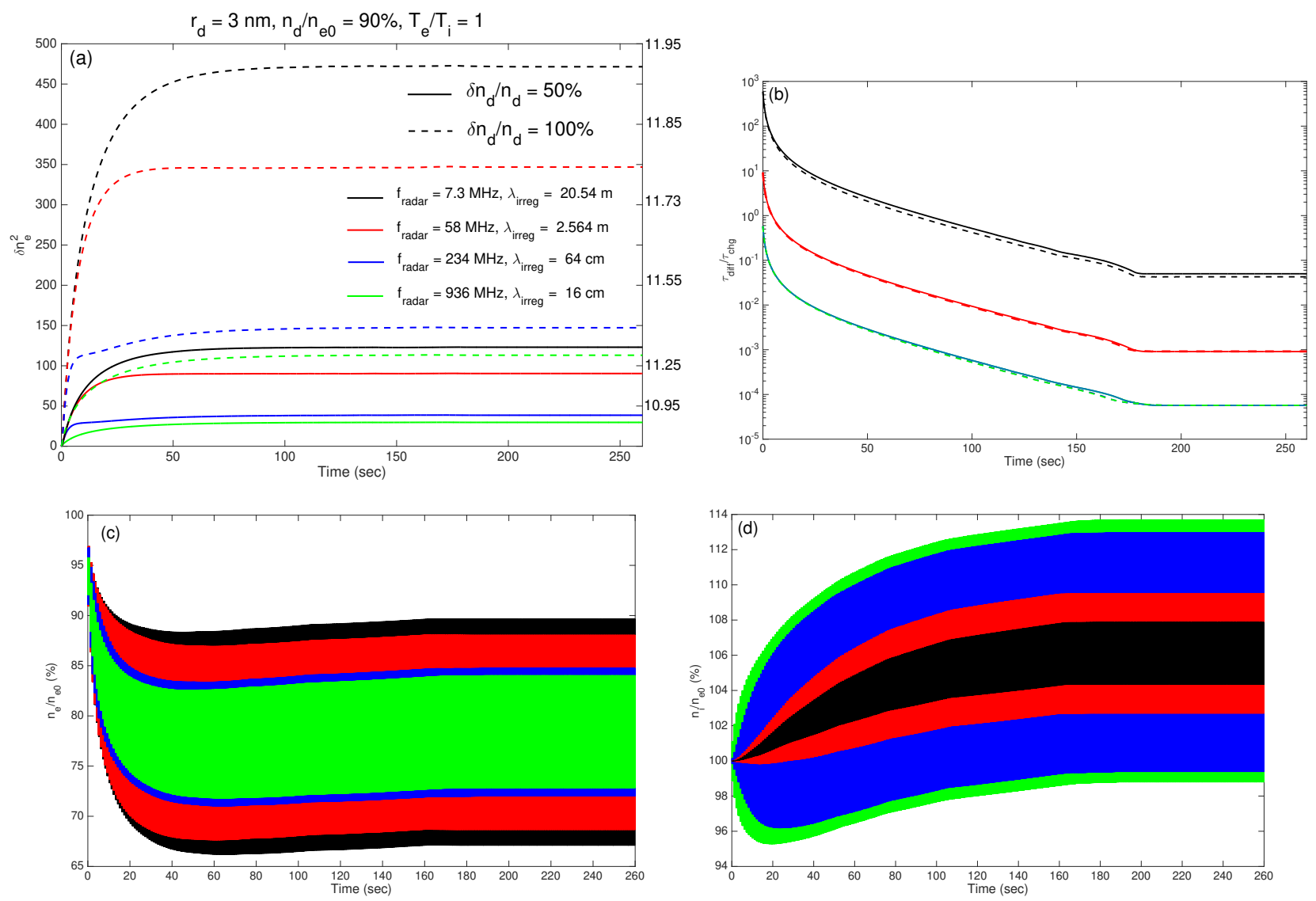

Figure 4. Numerical results associated with radar frequencies of $7.3 \mathrm{MHz}\left(\lambda_{\text {irreg }}=40.96 \mathrm{~m}\right), 58 \mathrm{MHz}\left(\lambda_{\text {irreg }}=5.12 \mathrm{~m}\right), 234 \mathrm{MHz}$ $\left(\lambda_{\text {irreg }}=1.28 \mathrm{~m}\right)$, and $936 \mathrm{MHz}\left(\lambda_{\text {irreg }}=32 \mathrm{~cm}\right)$. the mesospheric plasma parameters are $r_{d}=3 \mathrm{~nm}, n_{d} / n_{e 0}=90 \%, T_{e} / T_{i}=1$. The dashed lines correspond to the the initial dust fluctuation amplitude with respect to the background dust density $\delta n_{d 0} / \delta n_{d}=100 \%$, and solid lines denote $\delta n_{d 0} / \delta n_{d}=50 \%$. (a) shows the time evolution of electron density fluctuation amplitude squared ( $\left.\delta n_{e}^{2}\right)$, which is a proxy for the radar echoes. (b) represents the diffusion to charging time scales associated with each radar echoes. (c) the normalized electron density variation due to charging process on to the dust particles. (d) time evolution of the normalized ion density variation to the background electron density.

to smaller wavelength). Such effect could explain the coherent PMSE echoes observed at higher radar frequencies. While the increase of the Schmidt number to values much greater than unity (in the absence of dust particles) and extension of the viscous cut-off have been shown to some extent, no clear observational and computational modeling of PMSE at various frequency bands has been introduced.

This paper provides the first simultaneous observations of the PMSE source region with 4 ground-based radars. The radar frequencies $8 \mathrm{MHz}, 56 \mathrm{MHz}, 224 \mathrm{MHz}$, and $930 \mathrm{MHz}$ corresponding to Bragg scatter (coherent) electron density fluctuation wavelengths of $\lambda_{\text {irreg }}=40.96 \mathrm{~m}, 5.12 \mathrm{~m}, 1.28 \mathrm{~m}$, and $32 \mathrm{~cm}$, respectively, were employed. Such a wide range of radar 

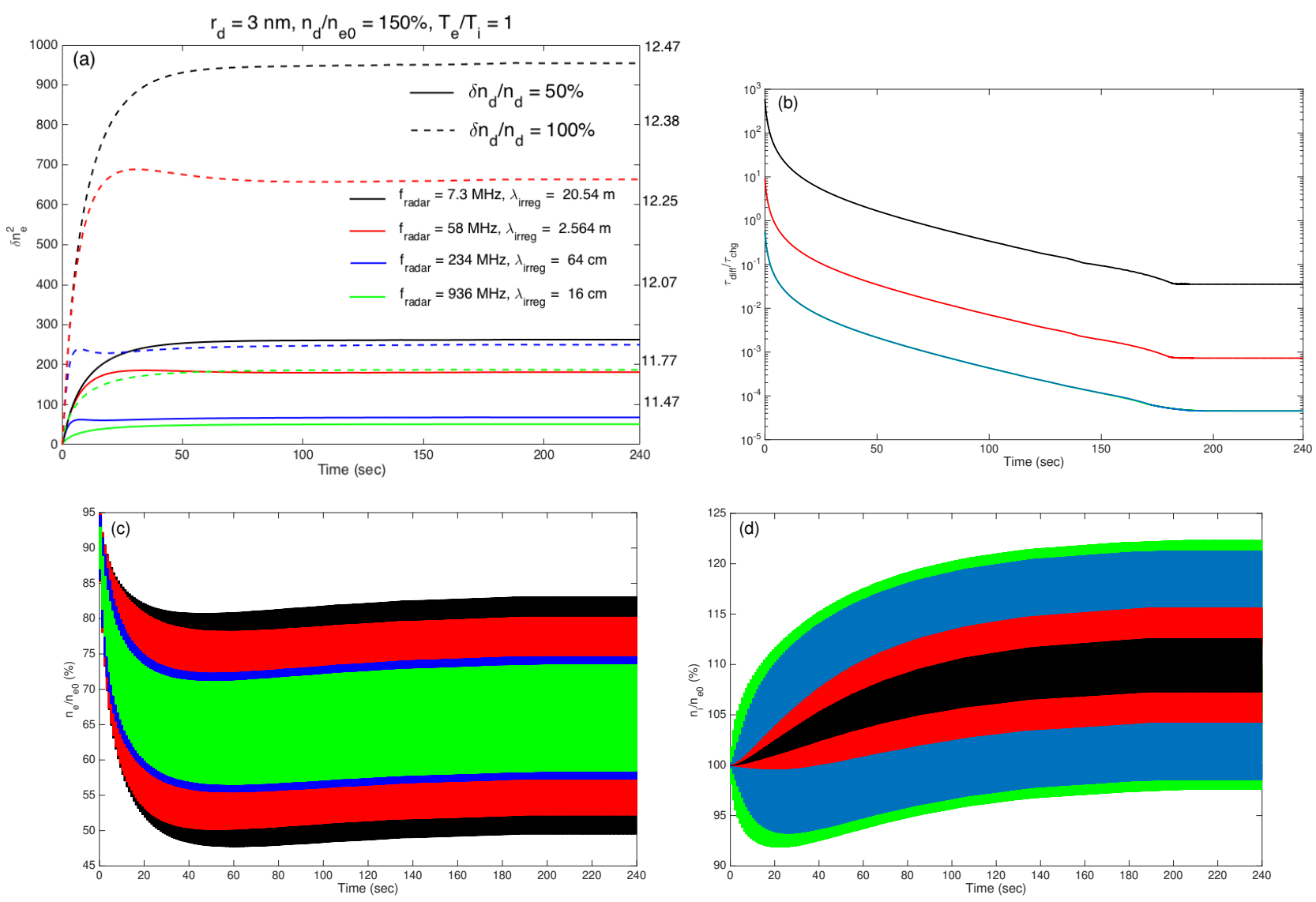

Figure 5. Similar to Figure 4 for $r_{d}=3 \mathrm{~nm}, n_{d} / n_{e 0}=150 \%, T_{e} / T_{i}=1$.

frequencies cover the irregularity wavelength regimes associated with the neutral air turbulence theory. This paper investigates the time evolution and steady state amplitude of the electron density fluctuation $\left(\delta n_{e}^{2}\right)$ in the presence of naturally perturbed mesospheric dust layers. The numerical calculation of $\delta n_{e}^{2}$ could be compared quantitively with the experimental observations of coherent radar echoes at various frequency band. Since the initial modulation of the dust density profile by the background neutral density profile has a great impact on the corresponding electron density fluctuation amplitude and radar echoes, two values of 0.5 and 1 are assumed for $\delta n_{d} / n_{d}$ parameter. Then plasma processes including electron/ion attachments to the background dust particles as well as electron density diffusion are allowed to develop and reach a steady state condition. Several background dusty plasma parameters including dust density and dust radius are allowed to vary in order to determine the corresponding amplitude of electron density fluctuation and the associated radar echoes at different wavelengths. Another important parameter that was investigated is the initial amplitude of dust density irregularities produced through neutral air turbulence $\left(\delta n_{d} / n_{d}\right)$. In the case of larger dust particles of the size of $10 \mathrm{~nm}$, the steady state condition is satisfied at much shorter time in comparison with $3 \mathrm{~nm}$ dust particles. In this case the stable fluctuation amplitude is achieved within $80 \mathrm{sec}$ from 

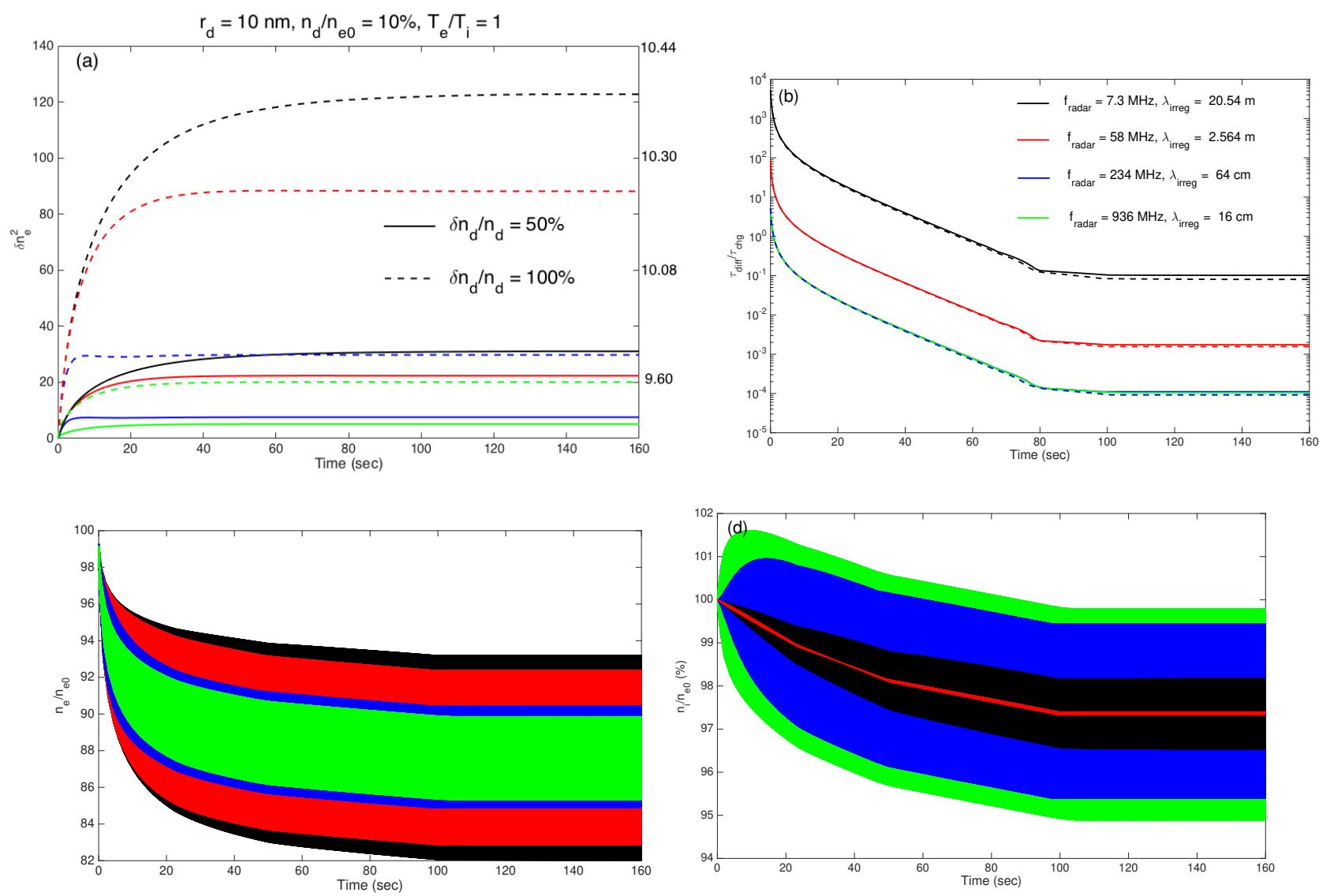

Figure 6. Similar to Figure 4 for $r_{d}=10 \mathrm{~nm}, n_{d} / n_{e 0}=10 \%, T_{e} / T_{i}=1$.

the equilibrium condition (quasi-neutral plasma in the absent of dust particles). This parameter manifests itself in the steady state amplitude of electron density fluctuations. An empirical relationship for the $\delta n_{e}^{2}$ with dust density with the same dust radius has been obtained using the numerical results. It has been shown that $\delta n_{e}^{2}$ increases by a factor of $\left(n_{d 2} / n_{d 1}\right)^{(6 / 5)}$.

One of the main features observed in the numerical simulations is that the electron density depletion is independent of the fluctuation wavelength and only varies with the background dust parameters. This effect validates the coherent scattering mechanism considered as the major source for plasma density irregularity generation. Therefore, it has been shown in this paper that the direct role of electron density depletion on corresponding radar echoes at various frequency bands is not possible, unlike the previous conclusion by Varney et al. (2011). In fact, the electron density fluctuation amplitude plays the major role in the coherent scattering process. The numerical results associated with various background dusty plasma is considered in order to investigate the 8, 56, 224, and $930 \mathrm{MHz}$ PMSEs.

In-situ rocket and radar observations have proved that neutral air turbulence by itself has a minor effect on the creation of small scale plasma irregularities in the PMSE region. The rocket measurements have shown a sharp cut off of fluctuations 

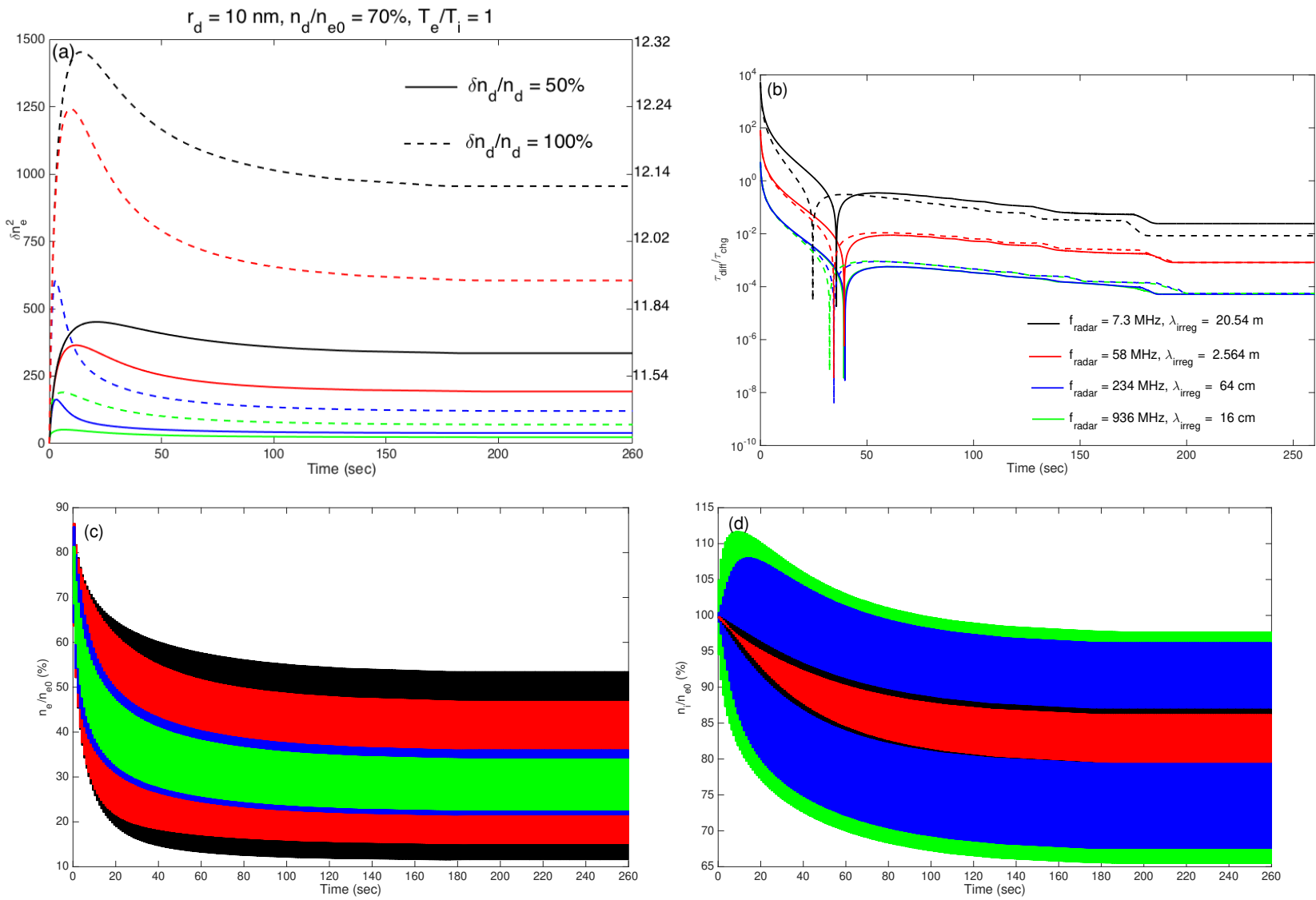

Figure 7. Similar to Figure 4 for $r_{d}=10 \mathrm{~nm}, n_{d} / n_{e 0}=70 \%, T_{e} / T_{i}=1$.

of the order of a few tens of meters ( $\sim 24 \mathrm{~m}$ ) (Rapp and Lubken, 2003). The long lifetime of PMSE below freezing altitude and even in the absence of neutral air turbulence have been attributed to frozen structure produced initially through turbulence advection and due to reduced diffusivity. The typical power spectrum of turbulence motion has two distinct parts. The first part represents a tracer with a wavenumber dependence of $k^{-5 / 3}$, which is known as the inertial subrange. The power spectrum continues with a second part where power spectrum is proportional to $k^{-1}$. Such spectral variation predicts a near zero power amplitude for Bragg scale of $\sim 5 \mathrm{~m}$ associated with $f_{r} \sim 56 \mathrm{MHz}$ due to dominant molecular diffusion. In general kinematic viscosity (diffusion of momentum) and turbulent energy dissipation rate (dissipation of turbulence energy to heat) are the two main parameters that affect the minimum scale of irregularities in the inertial subrange. The previous works on fossil turbulence have shown the importance of dust particle size as the main parameter that controls the viscosity and minimum steady scale of irregularities. Particles as large as $10 \mathrm{~nm}-15 \mathrm{~nm}$ around $85 \mathrm{~km}$ were proposed to justify the extended range of PMSE observations (Rapp and Lubken, 2003; La Hoz et al., 2006). The numerical simulations presented in this paper demonstrate the 
https://doi.org/10.5194/angeo-2020-81

Preprint. Discussion started: 8 December 2020

(c) Author(s) 2020. CC BY 4.0 License.

(c) (i)

major role of dust density along with dust radius as the two main parameters that determine the general behavior of turbulence power spectrum.
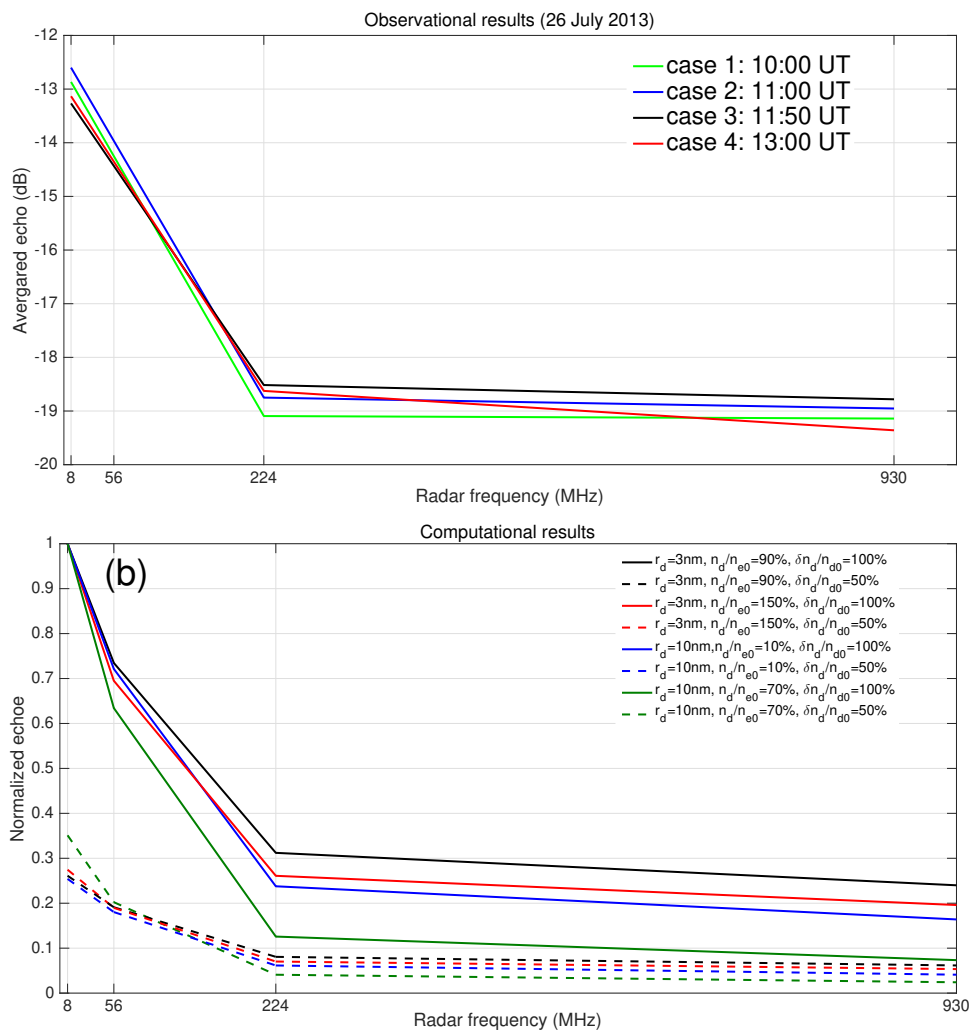

Figure 8. a) Averaged power echoes over the HF PMSE altitude range (in arbitrary unit) and associated with 26 July 2013 , in 4 cases at 10:00 UT, 11:00 UT, 11:50 UT, 13:00 UT. b) Numerical results associated with Figures 4-7 and corresponding to the variation of the radar echo strength with dust parameters.

The general correlation of the shape of the PMSE at different frequencies obtained through the observations reveal the existence of the plasma density fluctuations and associated structure over the same altitude range. The high resolution of observations of the turbulence region probed with with $8 \mathrm{MHz}, 56 \mathrm{MHz}, 224 \mathrm{MHz}$, and $930 \mathrm{MHz}$ radars show for the first time that fossil turbulence theory can be applied to the formation of PMSE. As discussed, while theoretical models fall short in predicting the dusty plasma parameters associated with the observed PMSEs, a computation model is used in this paper in order to determine the background parameters as well as provide an explanation for the non-existence (strong weakening) of PMSE between $224 \mathrm{MHz}$ and $930 \mathrm{MHz}$. According to the numerical results the dust radius $3 \mathrm{~nm}$ with density of 150 percent with respect to the background electron density $\left(n_{e}=2 \times 10^{9} \mathrm{~m}^{-3}\right)$ can produce radar echoes of the order of $12.5 \mathrm{~dB}\left(\log 10 N_{e}\right)$ for $f_{r}=7.3 \mathrm{MHz}$. This shows a close agreement with the experimental observations presented in Figure 1. Figure 8a presents the actual amplitude of radar echoes over the HF PMSE altitude range (80.91 km to $91.4 \mathrm{~km}$ ) and associated with 26 July 
2013, in 4 cases at 10:00 UT, 11:00 UT, 11:50 UT, 13:00 UT. Figure 8b also provides a summary of numerical results of radar echo associated with Figures 4-7 and corresponding to the variation of the radar echo strength with dust parameters. A close comparison between the two figures show a good correlation in regards to strength consistency as the radar frequency increases from $8 \mathrm{MHz}$ to $224 \mathrm{MHz}$ and $930 \mathrm{MHz}$. Such averaging is essential to determine the effectiveness as well as durability of the original turbulence in the presence of dusty plasma to generate coherent echoes from $8 \mathrm{MHz}$ to $930 \mathrm{MHz}$ (although it is very rare to observe PMSE at $930 \mathrm{MHz}$ ). Larger dust particles of $10 \mathrm{~nm}$ with smaller densities are also taken into account to examine the hypothesis of the role of larger dust particles in the PMSE formation. The numerical results has shown that for the similar dust parameters $\left(r_{d}\right.$ and $\left.n_{d}\right)$, the expected radar echoes $\left(\delta n_{e}^{2}\right)$ is very close for $224 \mathrm{MHz}$ and $930 \mathrm{MHz}$. It has been illustrated that the reduction of dust density fluctuation amplitude $\left(\delta n_{d} / n_{d}\right)$ by a factor of 2 would reduce the expected radar echoes by 1 $\mathrm{dB}$. The overall drop in the PMSE amplitude shows a similar drop as the $\delta n_{d} / n_{d}$ reduces from 1 to 0.5 for all dust parameters presented in Figures 4-7. Therefore, although according to the numerical results the estimated PMSE amplitude is similar for $224 \mathrm{MHz}$ and $930 \mathrm{MHz}$, the $\delta n_{d} / n_{d}$ plays a critical role in the steady state amplitude of irregularities and associated PMSE. This validates the non observation of UHF PMSE and can also be implemented to guide the previous theories used to predict the neutral air turbulence spectrum in the presence of dust particles.

Acknowledgements. EISCAT is an international association supported by research organizations in China (CRIRP), Finland (SA), Japan (NIPR and STEL), Norway (NFR), Sweden (VR), and the United Kingdom (NERC). The authors would like to thank Andrew Senior for his help in the data processing.

The data presented in this paper can be downloaded from the EISCAT online database at https://www.eiscat.se/scientist/data/ 


\section{References}

Batchelor, G. K. (1959), Small-scale variation of convected quantities like temperature in turbulent fluid. Part 1: General discussion and the case ofsmall conductivity,J. Fluid Mech.,5, 113-133.

Birdsall, C. K., and A. B. Langdon (1991), Plasma Physics Via Computer Simulation, McGraw-Hill, New York.

Chen, C., and Scales, W. A. (2005), Electron temperature enhancement effects on plasma irregularities associated with charged dust in the Earth's mesosphere, J. Geophys. Res., 110, A12313, doi:10.1029/2005JA011341.

Cho, J. Y. N., and Kelley, M. C. (1992), Enhancement of Thomson scatter by charged aerosols in the polar mesosphere: Measurements with a 1.29-GHz radar. Geophysical Research Letters, 19, 1097-1100.

Cho, J. Y. N. and Kelley, M. C. (1993), Polar mesospheric summer radar echoes: Observations and current theories, Rev. Geophys., 31, $243-265$.

Cho, J. Y. N. and Rottger, J. (1997), An updated review of polar mesospheric summer echoes: Observation, theory, and their relationship to noctilucent clouds and subvisible aerosols, J. Geophys. Res., 102, 2001-2020.

Christon, S. P., Hamilton, D. C., Plane, J. M. C., Mitchell, D. G., Grebowsky, J. M., Spjeldvik, W. N., and Nylund, S. R. (2017), Discovery of suprathermal ionospheric origin Fe+ in and near Earth's magnetosphere. Journal of Geophysical Research: Space Physics, 122, 11,175-11,200. https://doi.org/10.1002/2017JA024414

Ecklund, W. L., and Balsley, B. B. (1981), Long-term observations of the Arctic mesosphere with the MST radar at Poker Flat, Alaska. Journal of Geophysical Research, 86, 7775-7780.

Havnes, O, H Pinedo, C La Hoz, A Senior, TW Hartquist, MT Rietveld, and MJ Kosch (2015), A comparison of overshoot modeling with observations of polar mesospheric summer echoes at radar frequencies of 56 and $224 \mathrm{MHz}$, Ann. Geophys., 33(6), 737-747

Hill, R. J. (1978), Non neutral and quasi-neutral diffusion of weakly ionized multi constituent plasma, J. Geophys. Res.,83, 989-998.

Hill, R. J., Gibson-Wilde, D. E., Werne, J. A., and Fritts, D. C. (1999), Turbulence-induced fluctuations in ionization and application to PMSE, Earth Plan. Space, 51, 499-513.

La Hoz, C., O. Havnes, L. I. Næsheim, and D. L. Hysell (2006), Observations and theories of Polar Mesospheric SummerEchoes at a Bragg wavelength of 16 cm,J. Geophys. Res.,111, D04203, doi:10.1029/2005JD006044.

Lie-Svenson, O., Blix, T. A., and Hoppe, U. P. (2003), Modeling the plasma response to small-scale aerosol particle perturbations in the mesopause region, J. Geophys. Res., 108, 8442, doi:10.1029/2002JD002753.

Lubken, F., Hillert, W., Lehmacher, G., and von Zahn, U. (1993), Experiments revealing small impact of turbulence on the energy budget of the mesosphere and lower thermosphere, J. Geophys. Res., 98, 20 369-20 384.

Lubken, F.-J., Rapp, M., Blix, T., and Thrane, E. (1998), Microphysical and turbulent measurements of the Schmidt number in the vicinity of polar mesosphere summer echoes, Geophys. Res. Lett., 25, 893-896.

Lubken, F.-J., Rapp, M., and Hoffmann, P. (2002), Neutral air turbulence and temperatures in the vicinity of polar mesosphere summer echoes, J. Geophys. Res., 107 (D15), doi:10.1029/2001JD000915.

Mahmoudian, A., Scales, W. A., Kosch, M. J., Senior, A., and Rietveld, M. (2011), Dusty space plasma diagnosis using temporal behavior of polar mesospheric summer echoes during active modification. Annales Geophysicae, 29, 2169-2174. https://doi.org/10.5194/angeo-292169-2011. 
https://doi.org/10.5194/angeo-2020-81

Preprint. Discussion started: 8 December 2020

(c) Author(s) 2020. CC BY 4.0 License.

(c) (i)

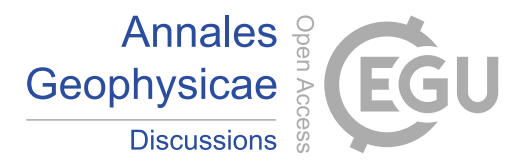

Mahmoudian, A., Mohebalhojeh, A. R., Farahani, M. M., Scales, W. A., and Kosch, M. (2017a), Remote sensing of mesospheric dust layers using active modulation of PMWE by high-power radio waves. Journal of Geophysical Research: Space Physics, 122, 843-856. https://doi.org/10.1002/2016JA023388.

Mahmoudian, A., Mohebalhojeh, A. R., Farahani, M. M., Scales (2017b), On the source of plasma density and electric field perturbations in PMSE and PMWE regions, Journal of the Earth and Space Physics, Vol. 42, No. 4, PP. 63-71

Mahmoudian, A., Senior, A., Scales, W. A., Kosch, M. J., and Rietveld, M. T. (2018), Dusty space plasma diagnosis using the behavior of polar mesospheric summer echoes during electron precipitation events. Journal of Geophysical Research: Space Physics, 123, 7697-7709. https://doi.org/10.1029/2018JA025395

Næsheim, L. I., O. Havnes, and C. La Hoz (2008), A comparison of polar mesosphere summer echo at VHF (224 MHz) and UHF (930 MHz) and the effects of artificial electron heating, J. Geophys. Res., 113, D08205, doi:10.1029/2007JD009245.

Rapp, M., and Lubken, F. J. (2004), Polar mesosphere summer echoes (PMSE): Review of observations and current understanding. Atmospheric Chemistry and Physics, 4, 2601-2633.

Robertson, S., Horanyi, M., Knappmiller, S., Sternovsky, Z., Holzworth, R., Shimogawa, M., Friedrich, M., Torkar, K., Gumbel, J., Megner, L., Baumgarten, G., Latteck, R., Rapp, M., Hoppe, U.-P., and Hervig, M. E.: Mass analysis of charged aerosol particles in NLC and PMSE during the ECOMA/MASS campaign, Ann. Geophys., 27, 1213-1232, doi:10.5194/angeo-27-1213-2009, 2009

Scales, W. (2004), Electron temperature effects on small-scale plasma irregularities associated with charged dust in the Earths mesosphere. IEEE Transactions on Plasma Science, 32, 724.

Scales, W. A., and Mahmoudian, A. (2016), Charged dust phenomena in the near Earth space environment. Reports on Progress in Physics, 79(10), 106802. https://doi.org/10.1088/0034-4885/79/10/106802

Senior, A., Mahmoudian, A., Pinedo, H., La Hoz, C., Rietveld, M. T., Scales, W. A., and Kosch, M. J. (2014), First modulation of high-frequency polar mesospheric summer echoes by radio heating of the ionosphere. Geophysical Research Letters, 41, 5347-5353. https://doi.org/10.1002/2014GL060703

Varney, R. H., Kelley, M. C., Nicolls, M. J., Heinselman, C. J., and Collins, R. L. (2011), The electron density dependence of polar mesospheric summer echoes, J. Atmos. Sol.-Terr. Phys., 73, 2153-2165, doi:10.1016/j.jastp.2010.07.020, 2011. 